\title{
Estimativa das contribuições dos sistemas anaeróbio lático e alático durante exercícios de cargas constantes em intensidades abaixo do $\mathrm{VO}$ $2 \max$
}

CDD. 20.ed. 796.027 796.073

\author{
Marcos David SILVA-CAVALCANTE*/** \\ Renata Gonçalves SILVA* \\ Rodrigo Poles URSO* \\ Rogério Carvalho SILVA* \\ Carlos Rafaell CORREIA-OLIVEIRA**** \\ Victor Gustavo Ferreira SANTOS ${ }^{* * * *}$ \\ Adriano Eduardo LIMA-SILVA** \\ Rômulo BERTUZZI*
}

*Escola de Educação Física e Esporte,

Universidade de São

Paulo.

**Faculdade de Nutrição,

Universidade Federal de

Alagoas.

\section{Resumo}

0 objetivo do estudo foi estimar as contribuições do metabolismo anaeróbio lático (MAL) e alático (MAA) em intensidades abaixo do consumo máximo de oxigênio ( $\left(\mathrm{V}_{2 \max }\right)$. Dez homens $(23 \pm 4$ anos, 176,4 \pm $6,8 \mathrm{~cm}, 72,4 \pm 8,2 \mathrm{~kg}, 12,0 \pm 4,5 \%$ de gordura corporal) realizaram um teste progressivo até a exaustão voluntária para identificação do $\dot{\mathrm{W}} \mathrm{O}_{2 \max }$ da potência correspondente ao $\dot{\mathrm{V}}_{2 \max }\left(\mathrm{WW}_{\mathrm{V}} \mathrm{O}_{\text {max }}\right)$ e do segundo limiar ventilatório $\left(L V_{2}\right)$. Na segunda e na terceira visita foram realizados seis testes de cargas constantes (três testes por sessão) com intensidades abaixo do $\dot{\mathrm{V}}_{\text {2max }_{\text {max }}}$. Houve uma predominância do MAL sobre o MAA durante os exercícios submáximos a partir da intensidade correspondente ao $\mathrm{LV}_{2^{\prime}}$ sendo significativamente maior em $90 \% \dot{\mathrm{V}}_{2 \max }(p<0,05)$. Dessa forma, esses resultados podem auxiliar treinadores a aplicarem cargas de treinamento adequadas aos seus atletas, de acordo com a exigência metabólica da competição.

PalaVRas-Chave: Exercícios sub- $\mathrm{v}_{2 \text { max }} ;$ Limiar ventilatório; Metabolismo anaeróbio alático; Metabolismo anaeróbio lático.

\section{Introdução}

A determinação da contribuição do metabolismo anaeróbio pode fornecer informações adicionais para a aplicação adequada da carga de treinamento e para a avaliação do desempenho físico. Acredita-se que durante os esforços realizados em intensidades acima do segundo limiar ventilatório $\left(\mathrm{LV}_{2}\right)$ uma parte da adenosina trifosfato (ATP) é ressintetizada pelo metabolismo anaeróbio alático (MAA) através da degradação da fosfocreatina (CP), e pelo metabolismo anaeróbio lático (MAL) por meio da degradação de glicogênio muscular, com subsequente formação de lactato ${ }^{1-2}$. Todavia, até o presente momento não existem relatos na literatura científica acerca da contribuição do MAA e MAL em intensidades abaixo do consumo máximo de oxigênio $\left(\tilde{W}_{2 \max }\right)$.
Estudos prévios têm demonstrado um aumento de aproximadamente $10 \%$ do déficit máximo acumulado de oxigênio após a suplementação com creatina monohidratada ${ }^{3}$ ou com cafeína ${ }^{4}$. Assumindo que o principal efeito ergogênico dessas substâncias está no acréscimo das concentrações de creatina e da atividade glicolítica intramuscular, é plausível pressupor que os valores do déficit máximo acumulado de oxigênio tenham sido modificados em razão do aumento da contribuição do MAA e MAL, respectivamente. Todavia, é importante destacar que esses estudos foram conduzidos em exercícios com alta intensidade, limitando a extrapolação desses achados para o exercício realizado em intensidades abaixo do $\stackrel{\mathrm{\vee}}{\mathrm{V}} \mathrm{2max}_{\text {ma }}$. 
Acredita-se que a contribuição dos sistemas anaeróbios possa ser estabelecida com mais precisão pela análise direta dos intermediários desses metabolismos, os quais são obtidos por meio de biópsia muscular antes e após o exercício físico 5 . Em virtude da característica invasiva dessa técnica e da dificuldade em se estimar a massa muscular envolvida na tarefa, alguns estudos têm empregado o componente rápido do excesso do consumo de oxigênio após o exercício $\left(\mathrm{EPOC}_{\text {rápido }}\right.$ ) o equivalente de oxigênio para o acúmulo de lactato sanguíneo $\left(\mathrm{E}_{\text {[LA] }}\right)$ na determinação da contribuiçãao do MAA e MAL, respectivamente ${ }^{6-8}$. Esses métodos assumem que, durante a fase inicial do período de recuperação, o restabelecimento dos estoques da CP ocorre por processos metabólicos que dependem do consumo de oxigênio $\left(\bar{V}_{2}\right)^{6-7,9-12}$. Além disso, ao observarem que as concentrações sanguíneas de lactato aumentavam

\section{Método}

\section{Amostra}

Dez indivíduos do sexo masculino $(23 \pm 4$ anos, $176,4 \pm 6,8 \mathrm{~cm}, 72,4 \pm 8,2 \mathrm{~kg}, 12,0 \pm 4,5 \% \mathrm{de}$ gordura corporal), fisicamente ativos, aparentemente saudáveis e já familiarizados com exercício exaustivo participaram voluntariamente desse estudo após a leitura e assinatura do termo de consentimento livre e esclarecido. Todos os sujeitos estavam isentos de tratamentos farmacológicos, não eram fumantes e estavam livres de qualquer tipo de distúrbio neuromuscular ou cardiovascular. Os procedimentos adotados no presente estudo foram previamente aprovados pelo Comitê de Ética em Pesquisa da Escola de Educação Física e Esporte da Universidade de São Paulo.

\section{Desenho experimental}

Todos os sujeitos foram submetidos a três sessões experimentais, as quais tiveram o intervalo mínimo de 72 horas e máximo de três semanas. Na primeira sessão, os sujeitos realizaram um teste progressivo até a exaustão voluntária em um cicloergômetro para a mensuração do ${ }^{\mathrm{V}} \mathrm{O}_{2 \max }$. Além disso, foi mensurada a potência externa correspondente ao $\mathrm{LV}_{2}\left(\mathrm{WLV}_{2}\right)$ e a potência correspondente ao $\mathrm{W}_{2 \text { max }}\left(\mathrm{W}_{\mathrm{W}} \mathrm{O}_{2 \max }\right)$. Na segunda e na terceira sessões os sujeitos foram submetidos a seis testes de cargas constantes (três testes por sessão) com intensidades abaixo do ${ }^{\circ} \mathrm{O}_{2 \max }$. A ordem de forma proporcional a potência metabólica, DI Prampero e FerRetTi ${ }^{7}$ sugeriram que é possível expressar em equivalente de oxigênio $\left(\mathrm{O}_{2}\right)$ a energia oriunda do acúmulo de lactato no sangue $\left(\mathrm{E}_{[[\mathrm{LA}]}\right)$.

Nesse sentido, considerando que o conhecimento da contribuição dos metabolismos anaeróbios podem ajudar treinadores na prescrição do treinamento, e que não existem estudos apontando a contribuição desses sistemas em exercícios de intensidades abaixo do $\dot{\mathrm{V}} \mathrm{O}_{2 \max }$, o objetivo do presente estudo foi estimar as contribuições do MAL e MAA em função da intensidade do exercício físico de carga constante em intensidades abaixo do $\mathrm{VO}_{2 \max }$. Uma vez que existe um incremento exponencial na produção de lactato em intensidades acima do $\mathrm{LV}_{2}{ }^{13}$, a nossa hipótese era que a contribuição do MAL para o total de energia produzida anaerobicamente seria maior em intensidades acima do $\mathrm{LV}_{2}$ em comparação ao MAA.

das sessões 2-3, bem como a dos testes sub- $\mathrm{V}_{2 \max }$ dentro da sessão, foi estabelecida aleatoriamente. Os testes foram realizados com a temperatura ambiente semelhante $\left(20-24^{\circ} \mathrm{C}\right)$ e duas horas após a última refeição. Os sujeitos foram solicitados a não praticarem exercícios físicos extenuantes e a não ingerirem álcool nas 48 horas que antecederam as coletas dos dados. No intuito de evitar possíveis influências ergogênicas ${ }^{4} \mathrm{e}$ do ritmo cicardiano ${ }^{14}$, todos os testes foram realizados no mesmo período do dia e os sujeitos foram instruídos a não consumirem nas 48 horas que precediam os testes substâncias que possuíssem cafeína.

\section{Determinação do consumo máximo de oxigênio e do limiar ventilatório}

O teste incremental até a exaustão voluntária foi realizado em um cicloergômetro eletromagnético de membros inferiores (Godart-Holland, Lannoy). A altura do selim foi individualmente ajustada, tendo como referência a extensão quase total dos joelhos dos sujeitos em um ciclo completo do pedal. Antes do início do teste, os participantes permaneceram cinco minutos sentados no cicloergômetro para o estabelecimento da linha de base do $\dot{\mathrm{V}} \mathrm{O}_{2}\left(\dot{\mathrm{V}} \mathrm{O}_{2 \mathrm{LB}}\right)$, a qual foi determinada a partir da média aritmética do $\dot{\mathrm{V}} \mathrm{O}_{2}$ nos 30 segundos finais desse período. Após o aquecimento de três minutos com apenas 
a resistência inercial do equipamento, os sujeitos se exercitaram com o ritmo em $60 \mathrm{rpm}$ e com o incremento da intensidade de $30 \mathrm{~W} \cdot \mathrm{min}^{-1}$. O teste foi interrompido quando a cadência do pedal era menor que $50 \mathrm{rpm}$. Durante todo o teste as trocas gasosas e a frequência cardíaca (FC) foram mensuradas respiração a respiração e a cada batimento, respectivamente. Amostras de sangue foram coletas imediatamente após o teste, no segundo e no terceiro minutos da recuperação para mensuração das concentrações sanguíneas lactato de pico ([La- $]$ pico), ao passo que a frequência cardíaca máxima $\left(\mathrm{FC}_{\max }\right)$ foi estabelecida pelo maior valor medido ao final do teste. $\mathrm{O} \mathrm{VO}_{2 \max }$ foi determinado a partir da obtenção de, pelo menos, três dos seguintes critérios: exaustão do sujeito, o aumento do $\mathrm{WO}_{2}$ menor que $2,1 \mathrm{ml}^{\mathrm{kg}}{ }^{-1} \mathrm{~min}^{-1}$ mediante o incremento da intensidade, a razão de trocas respiratórias maior que 1,10 , concentração de lactato sanguíneo após o teste maior que $8,0 \mathrm{mmol}^{-1} \mathrm{l}^{-1}$, atingir a frequência cardíaca máxima $\left(\mathrm{FC}_{\max }\right)$ predita pela idade $(220 \text {-idade })^{15}$. A $\mathrm{WUO}_{2 \max }$ foi estabelecida como a potência externa máxima em que o ${ }^{2} \mathrm{O}_{2 \max }$ foi alcançado. $\mathrm{O} \mathrm{LV}_{2}$ foi estabelecido visualmente mediante o segundo ponto de inflexão da curva da ventilação, com concomitante queda da pressão parcial de dióxido de carbono ${ }^{16}$.

\section{Testes com cargas constantes}

O cicloergômetro, o ajuste da altura do selim, o ritmo do pedal, o aquecimento, o critério de interrupção e a mensuração do $\mathrm{v}_{2}$ nos exercícios com cargas constantes foram idênticos aos empregados no teste progressivo até a exaustão. Os sujeitos se exercitaram por 10 minutos, ou até a exaustão voluntária, em seis testes de intensidades abaixo da $\mathrm{W}^{\mathrm{W}} \mathrm{O}_{2 \max }\left(40-90 \% \mathrm{~W}^{\mathrm{W}} \mathrm{O}_{2 \max }\right)$. O período de recuperação entre essas tarefas foi de aproximadamente 10 minutos, ou até atingir o valor individual do $\dot{\mathrm{V}} \mathrm{O}_{2 \mathrm{LB}}$. A média do $\dot{\mathrm{V}} \mathrm{O}_{2}$ no último minuto dessas tarefas foi utilizada para representar o valor de pico do ${ }^{\mathrm{V}} \mathrm{O}_{2}$ nessas tarefas. Além disso, amostras contendo $25 \mu \mathrm{l}$ de sangue foram coletadas do lóbulo da orelha antes de cada teste ([ $\left.\mathrm{La}^{-}\right]$ repouso), imediatamente após o término, no terceiro, no quinto e no sétimo minutos de recuperação para a mensuração das concentrações sanguíneas de lactato [La] . O maior valor após o exercício foi utilizado para representar o lactato de pico.

\section{Coleta dos dados fisiológicos}

$\mathrm{O} \dot{\mathrm{V}} \mathrm{O}_{2}$ foi mensurado continuamente por meio de um analisador de gases portátil $\left(\mathrm{K}_{4} \mathrm{~b}^{2}\right.$ Cosmed, Roma, Itália), ao passo que a FC foi medida por um cardiofrequencímetro (Polar, Kempele, Finlândia) acoplado a esse equipamento. Uma máscara de silicone com baixa resistência que mantém o nariz e a boca cobertos (Hans Rudolph, Kansas City, USA) foi empregada na coleta do ar utilizado nas análises da ventilação, das frações do oxigênio e do dióxido de carbono. Antecedendo cada sessão de coleta de dados, o analisador de gases foi calibrado utilizando o ar ambiente e com um gás de composição conhecida $\left(20,9 \%\right.$ de $\mathrm{O}_{2}$ e $5 \%$ de $\mathrm{CO}_{2}$ ). A calibração do fluxo de ar foi realizada com uma seringa com volume de três litros (Quinton Instruments, Seattle, WH). A calibragem do tempo de atraso para a análise da amostra de ar expirada foi realizada de acordo com as especificações do fabricante (K4b2 instruction manual). Esse tempo de atraso é de aproximadamente $500 \mathrm{~ms}$ e automaticamente considerado nos cálculos das trocas gasosas. As [ $\left.\mathrm{La}^{-}\right]$foram determinadas por meio de um analisador automático de lactato (Yellow Springs 1500 Sport, Ohio, USA), o qual foi calibrado a cada 10 análises, tendo como referência a concentração de lactato de $5 \mathrm{mmol} \cdot \mathrm{l}^{-1}$.

\section{Cálculos}

Em todos os testes de carga constante a contribuição do MAA foi assumida como sendo o EPOC $_{\text {rápido }}{ }^{17}$. Conforme descrito previamente por ÖZYENER et al. ${ }^{19}$, a análise da resposta cinética "off" do $\dot{\mathrm{V}} \mathrm{O}_{2}$ nas intensidades abaixo do $\mathrm{LV}_{2}$ foi ajustada por um modelo monoexponencial (Equação 1), ao passo que nas intensidades acima do $\mathrm{LV}_{2}$ foi aplicado um modelo biexponencial (Equação 2) (Origin, Microcal, USA). Assim, a contribuição do sistema anaeróbio alático pode ser determinada pela integração da parte exponencial do componente rápido do excesso do consumo após o exercício (Equação 3).

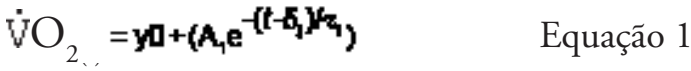

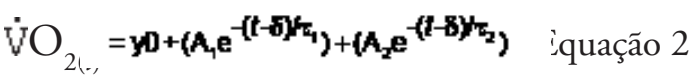

$$
\begin{aligned}
& \mathrm{MAA}=\mathrm{A}_{1} \cdot \tau_{1} \quad \text { Equação } 3
\end{aligned}
$$


Onde ${ }_{1} \mathrm{e}_{2}$ denotam, respectivamente, o componente rápido e lento do excesso do consumo de oxigênio após o exercício; $y 0=$ linha de base; $A, \tau$ e $\delta$ são referentes à amplitude $\mathrm{em} \mathrm{ml} \cdot \mathrm{min}^{-1}$, à constante de tempo (s) e ao tempo de atraso (s), respectivamente.

A contribuição do MAL foi estimada assumindo que $1 \mathrm{mmol}^{-1} \mathrm{l}^{-1}$ dos $\Delta\left[\mathrm{La}^{-}\right]$no exercício sub- $\mathrm{V}^{\top} \mathrm{O}_{2 \max }$ equivale ao valor fixo de $3 \mathrm{ml}$ de $\mathrm{O}_{2} \mathrm{~kg}^{-1}$ de massa corporal ${ }^{7}$.

\section{Análises estatísticas}

Todas as análises foram feitas utilizando o "software" SPSS (versão 13.0, Chicago, USA). A

\section{Resultados}

As variáveis analisadas no teste progressivo até a exaustão estão presentes na TABELA 1.

A FIGURA 1 apresenta um exemplo típico da resposta do $\mathrm{VO}_{2}$ durante essas tarefas.

A TABELA 2 apresenta os valores do pico do "ن duração dos testes sub- $\mathrm{W}^{\mathrm{W}} \mathrm{O}_{2 \max }$. A partir da intensidade de $50 \% \mathrm{~V}_{2 \max }$ foi constatado o aumento significativo do $\mathrm{WO}_{2}(\mathrm{~F}=152,17 ; \mathrm{p}<0,001)$ e das [La $\left.{ }^{-}\right](\mathrm{F}=53,81 ; \mathrm{p}<0,001)$, quando comparados à intensidade de $40 \% \mathrm{VO}_{2 \max }$. A FC teve o aumento significativo apenas a partir da intensidade de $60 \%$ distribuição dos dados foi verificada por meio do teste de Shapiro-Wilk e apresentaram distribuição normal. Os dados foram reportados como médias e desvios padrão. A análise de variância com medidas repetidas a um fator (intensidade) seguido pelo teste para comparaçōes múltiplas de Bonferroni foi utilizada na comparação das variáveis mensuradas nos testes sub- $\mathrm{VO}_{2 \max }$. Para comparar a contribuição do MAL e MAA em cada intensidade, foi empregado o teste t. A esfericidade dos dados foi verificada previamente pelo teste de Mauchly. O nível de significância adotado foi de $5 \%(\mathrm{p}<0,05)$.

TABELA 1 - Variáveis analisadas no teste progressivo até a exaustão $(\mathrm{n}=10)$.

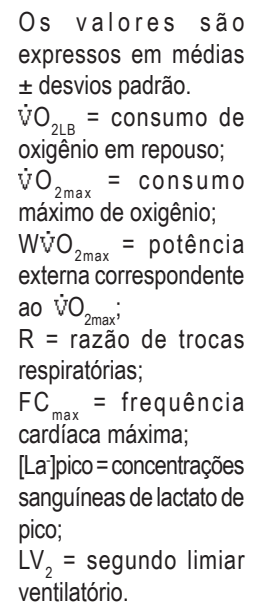

$\mathrm{W}^{\circ} \mathrm{O}_{2 \max }(\mathrm{F}=52,12 ; \mathrm{p}<0,001)$, ao passo que a primeira intensidade em que um sujeito não conseguiu completar 10 minutos de exercício foi $70 \%$ WTO

A FIGURA 2 apresenta o comportamento das contribuições percentuais do MAL e MAA durante o exercício realizado abaixo da $\mathrm{W}^{\top} \mathrm{O}_{2 \max }$. A transição da predominância do MAA para o MAL na composição do metabolismo anaeróbio se inicia em, aproximadamente, $70 \%$ da $\mathrm{W}^{\circ} \mathrm{W}_{2 \max }$. Essa carga corresponde à média do valor da intensidade do $\mathrm{LV}_{2}$ (TABELA 1).

\begin{tabular}{|c|c|}
\hline$\dot{\mathrm{W}} \mathrm{O}_{2 \mathrm{LB}}\left(\mathrm{l} \cdot \mathrm{min}^{-1}\right)$ & $0,28 \pm 0,57$ \\
\hline$\dot{\mathrm{V}} \mathrm{O}_{2 \max }\left(1 \cdot \min _{-1}\right)$ & $3,0 \pm 0,5$ \\
\hline $\mathrm{W}^{\prime} \mathrm{O}_{2 \max }\left(\mathrm{ml}^{\prime} \mathrm{kg}^{-1} \cdot \mathrm{min}^{-1}\right)$ & $41,5 \pm 5,7$ \\
\hline $\mathrm{W}^{\top} \mathrm{O}_{2 \max }(\mathrm{W})$ & $250 \pm 40$ \\
\hline $\mathrm{R}$ & $1,29 \pm 0,09$ \\
\hline $\mathrm{FC}_{\max }(\mathrm{bpm})$ & $179 \pm 9$ \\
\hline$\left[\mathrm{La}^{-}\right]$pico $\left(\mathrm{mmol}^{-1} \mathrm{l}^{-1}\right)$ & $10,28 \pm 1,42$ \\
\hline Potência no $\mathrm{LV}_{2}(\mathrm{~W})$ & $180 \pm 30$ \\
\hline Potência no $\mathrm{LV}_{2}\left(\% \mathrm{~W}^{\mathrm{T}} \mathrm{O}_{2 \max }\right)$ & $72 \pm 8$ \\
\hline
\end{tabular}




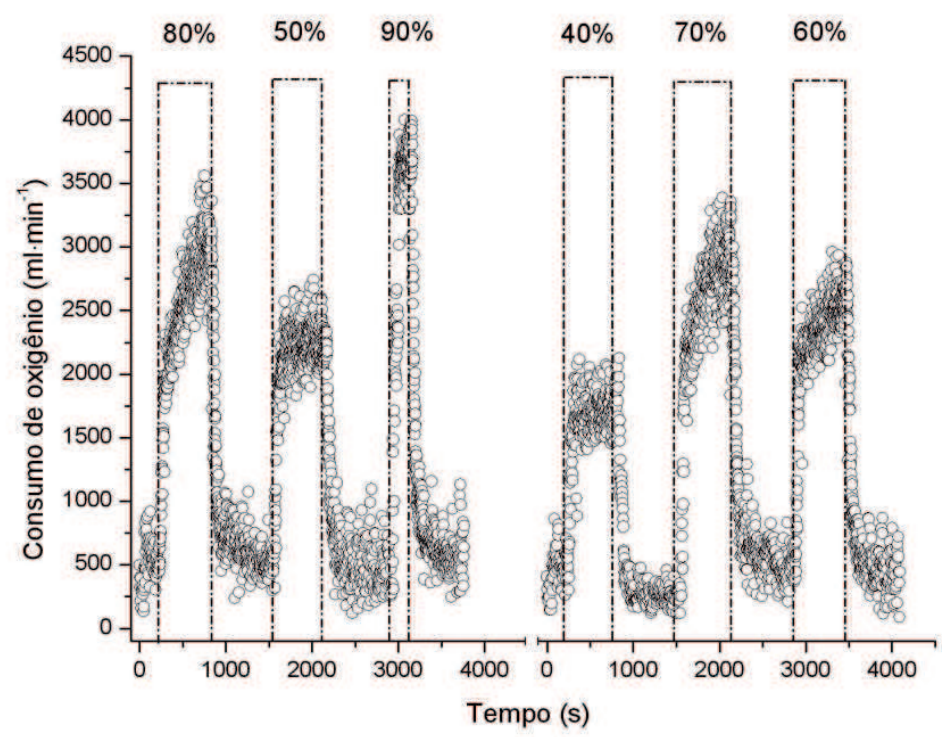

As linhas pontilhadas marcam o início e o término do exercício.

FIGURA 1 - Exemplo do comportamento típico do consumo de oxigênio nos testes abaixo da potência externa correspondente ao consumo máximo de oxigênio (\%) em duas sessões experimentais distintas.

TABELA 2 - Respostas do pico do consumo de oxigênio, da frequência cardíaca, das concentrações sanguíneas de lactato e do tempo de duração do exercício executado nas intensidades abaixo da potência externa correspondente ao consumo máximo de oxigênio $(n=10)$.

\begin{tabular}{lcccc}
\hline $\begin{array}{l}\text { Intensidade } \\
\left(\% \mathrm{~W}_{\mathbf{W}} \mathrm{O}_{2 \max }\right)\end{array}$ & $\begin{array}{c}\dot{\mathrm{WO}} \\
\left(1 \cdot \mathrm{min}^{-1}\right)\end{array}$ & $\begin{array}{c}\mathrm{FC} \\
(\mathrm{bpm})\end{array}$ & $\begin{array}{c}{\left[\mathrm{La}^{-}\right]} \\
\mathrm{mmol}^{-1}\end{array}$ & $\begin{array}{c}\text { Duração } \\
(\mathrm{s})\end{array}$ \\
\hline $40 \%$ & $1,71 \pm 0,18$ & $123 \pm 8$ & $2,59 \pm 1,45$ & $600 \pm 0$ \\
$50 \%$ & $2,19 \pm 0,22^{\mathrm{d}}$ & $145 \pm 9$ & $4,84 \pm 1,89^{\mathrm{d}}$ & $600 \pm 0$ \\
$60 \%$ & $2,51 \pm 0,27^{\mathrm{c}}$ & $155 \pm 18^{\mathrm{c}}$ & $6,97 \pm 1,67^{\mathrm{c}}$ & $600 \pm 0$ \\
$70 \%$ & $2,71 \pm 0,25^{\mathrm{b}}$ & $175 \pm 10^{\mathrm{b}}$ & $8,59 \pm 1,30^{\mathrm{c}}$ & $577 \pm 72$ \\
$80 \%$ & $2,97 \pm 0,31^{\mathrm{e}}$ & $179 \pm 9^{\mathrm{b}}$ & $10,22 \pm 1,30^{\mathrm{b}}$ & $444 \pm 139$ \\
$90 \%$ & $3,17 \pm 0,31^{\mathrm{a}}$ & $177 \pm 10^{\mathrm{a}}$ & $9,90 \pm 1,21^{\mathrm{b}}$ & $306 \pm 128$ \\
\hline
\end{tabular}

Os valores são expressos em médias \pm desvios padrão.

a $=$ maior que $40,50,60$, 70 e $80 \%$;

b $=$ maior que 40,50 e $60 \%$;

$c=$ maior que 40 e $50 \%$;

d $=$ maior que $40 \%$;

$\mathrm{e}=40,50,60$ e $70 \%$.

Nível de significância $p<0,05$.

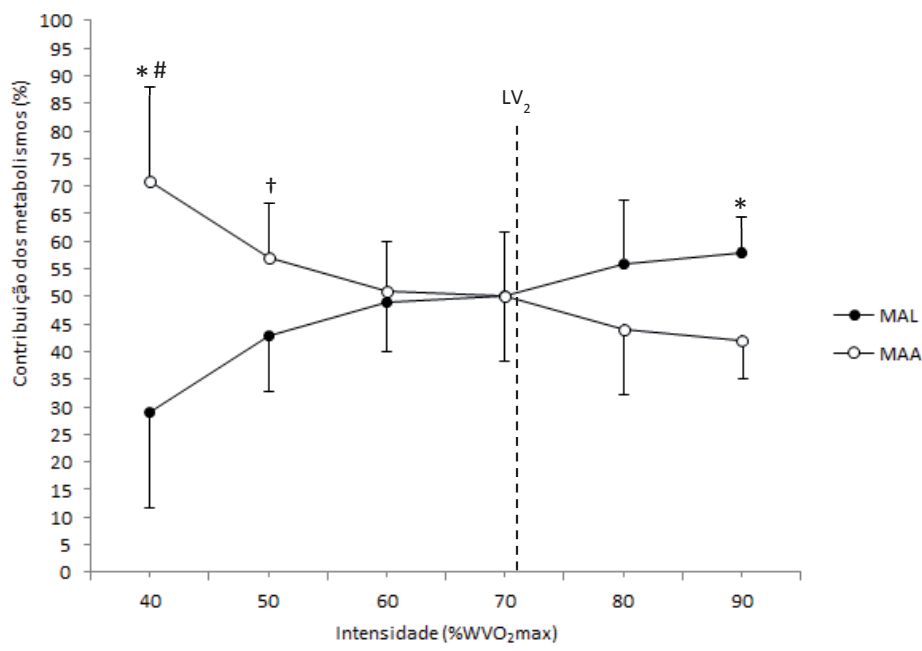

A linha pontilhada delimita o início do segundo limiar ventilatório $\left(\mathrm{LV}_{2}\right)$.

* Diferença entre o MAL e MAA $(p<0,05)$.

\#Diferente de 60, 70, 80 e $90 \% \mathrm{WVO}_{2 \max }$ em MAL e MAA $(p<0,05)$.

tDiferente de $90 \% \mathrm{WVO}_{2 \max }$ em MAL e MAA $(p<0,05)$

FIGURA 2 - Exemplo do comportamento típico do consumo de oxigênio nos testes abaixo da potência externa correspondente ao consumo máximo de oxigênio (\%) em duas sessões experimentais distintas. 


\section{Discussão}

Acredita-se que a determinação das contribuições dos metabolismos energéticos pode fornecer informaçōes importantes para aprimorar a carga de treinamento e para a avaliação do desempenho físico. Todavia, até o presente momento, os estudos prévios realizaram essas estimativas em intensidades acima da potência aeróbia máxima ${ }^{4,17}$. Para o melhor do nosso conhecimento, esse é o primeiro estudo a analisar as contribuições do MAA e do MAL durante exercícios com intensidades abaixo da potência aeróbia máxima. O principal achado do presente estudo foi que a predominância do MAL sobre o MAA durante os exercícios submáximos ocorreu a partir da intensidade correspondente ao $\mathrm{LV}_{2}$.

Evidências mais recentes têm indicado que a degradação da $\mathrm{CP}$ não ocorre apenas para a manutenção da oferta da ATP durante o exercício de alta intensidade e de curta duração ${ }^{20-21}$. Assim, o comportamento das contribuiçōes do MAA e do MAL durante os exercícios de cargas constantes reforça as hipóteses que foram estabelecidas acerca da relação entre o MAA e o metabolismo oxidativo no início e durante o exercício físico de intensidade moderada. Alguns trabalhos realizados com o interesse de compreender a dinâmica da resposta cinética "on" do $\mathrm{VO}_{2}$, mais especificamente a fase II (ou componente primordial), têm demonstrado que a ativação do metabolismo oxidativo é dependente da degradação da ATP e da CP na fase inicial do exercício físico ${ }^{22-23}$. Possivelmente, o aumento das concentrações de ADP, Pi e da creatina na forma livre seria um dos mecanismos responsáveis pela regulação da respiração mitocondrial ${ }^{21-22,24}$.

Isso é possível porque a $\mathrm{CP}$ tem a capacidade de atuar como um "transportador de energia" mediante o sistema de lançadeira de creatina ("creatine shuttle" ${ }^{20-21}$. Durante a contração muscular de baixa intensidade, a redução das concentraçōes da ATP é amenizada pela ativação da isoforma extramitocondrial da creatina quinase, a qual possibilita ressintetizar a ATP mediante a degradação da CP. Uma vez que a membrana externa da mitocôndria é permeável à creatina, o seu aumento no espaço entre as membranas mitocondriais resulta na produção de ADP com subsequente ressíntese de $\mathrm{CP}$, graças ao aumento da atividade da isoforma mitocondrial da creatina quinase. Posteriormente, a CP pode ser deslocada para o citoplasma no intuito de ressintetizar a ATP sarcoplasmática ${ }^{25}$.
Embora a sua origem tenha sido objetivo de recentes discussões ${ }^{26}$, é incontestável que o aumento das concentrações citoplasmáticas de $\mathrm{H}^{+}$deve-se direta ou indiretamente à ativação do MAL. Em um estudo realizado com músculos isolados de ratos, constatou-se que o aumento excessivo do $\mathrm{Pi}(20$ $\mathrm{mM}$ ) e a queda acentuada do $\mathrm{pH}$ (6.6) são capazes de reduzir a respiração mitocondrial ${ }^{24}$. Em outras palavras, demonstrou-se que pequenas mudanças nesses parâmetros podem ativar a fosforilação oxidativa, ao passo que grandes mudanças podem afetar a função mitocondrial. Teoricamente, isso se deve à ativação da isoforma extramitocondrial da creatina quinase ser gravemente prejudicada pelas elevadas concentrações de Pi e de $\mathrm{H}^{+24}$. Por sua vez, o conceito do limiar anaeróbio foi desenvolvido para estimar a intensidade do esforço na qual a energia fornecida pelo metabolismo aeróbio é complementada pelo MAL ${ }^{27}$. Logo, é atraente suspeitar que, devido ao aumento acentuado das concentraçōes de $\mathrm{H}^{+}$nas intensidades acima do $\mathrm{LV}_{2}$, haveria uma redução da respiração mitocondrial em virtude da redução no funcionamento do sistema de lançadeira de creatina. Como consequência, ocorreria a transição da predominância da contribuição percentual do MAA para o MAL. Entretanto, essa inferência deve ser apreciada com cautela, pois em algumas circunstâncias, as [ $\left.\mathrm{La}^{-}\right]$antes do segundo e do terceiro testes de uma dada sessão experimental eram visualmente maiores que as [ $\left.\mathrm{La}^{-}\right]$de repouso. De certa forma, isso pode ter influenciado os cálculos da contribuição do MAL nessas tarefas.

O método utilizado no presente estudo para quantificar as contribuições dos sistemas anaeróbio apresenta limitações. Uma vez que o lactato plasmático não representa o total de lactato produzido pelos músculos durante o exercício. Dessa forma, os resultados do presente estudo obtidos por meio desse método representa uma estimativa da contribuição do sistema anaeróbio. No entanto, vale destacar que esse método é o único disponível até o presente momento capaz de distinguir a contribuição do MAL e do MAA ${ }^{28-29}$.

Em resumo, os dados do presente estudo indicam a existência de uma predominância da contribuição do MAL sobre o MAA em intensidades acima do limiar ventilatório. Nesse sentido, esses resultados podem auxiliar treinadores a aplicarem cargas de treinamento adequadas aos seus atletas, de acordo com a exigência metabólica da competição. 


\section{Abstract}

Estimation of contributions of the anaerobic lactic and alactic systems during constant-load exercises at intensities below the $\mathrm{VO}_{2 \max }$

The purpose this study was that estimated contributions of the anaerobic lactic (MAL) and alactic (MAA) metabolism during constant load exercises at intensities below the maximal oxygen capacity uptake ( $\left(\mathrm{W} \mathrm{O}_{2 \text { max }}\right)$. Ten males ( $23 \pm 4$ years, $176.4 \pm 6.8 \mathrm{~cm}, 72.4 \pm 8.2 \mathrm{~kg}, 12.0 \pm 4.5 \%$ of fat body) performed in the first visit a progressive test until exhaustion to identification of ${ }^{\mathrm{V}} \mathrm{O}_{2 \max }$ power output corresponding to the $\mathrm{VO}_{2 \max }\left(\mathrm{WWO}_{2 \max }\right)$ and second ventilatory threshold $\left(\mathrm{LV}_{2}\right)$. On the second and third visit, the participants performed six constant workload tests (3 per session) with intensities below $\mathrm{V}_{2 \max }$. There was a predominance of MAL about MAA during the exercises sub-maximal from intensity corresponding to the $\mathrm{LV}_{2^{\prime}}$ being significantly higher at $90 \% \mathrm{WO}_{2 \max }(\mathrm{p}<0.05)$. Thus, these results may help coaches to implement training loads appropriate to their athletes, according to the metabolic demand of the competition.

KEY WORDS: SUb- $\mathrm{WO}_{2 \max }$ work load; Ventilatory threshold; Anaerobic alactic metabolism; Anaerobic lactic metabolism.

\section{Referências}

1. Vøllestad NK, Vaage O, Hermansen L. Muscle glycogen depletion patterns in type I and subgroups of type II fibres during prolonged severe exercise in man. Acta Physiol Scand. 1984;122:433-41.

2. Marsh GD, Paterson DH, Thompson RT, Driedger AA. Coincident thresholds in intracellular phosphorylation potential and $\mathrm{pH}$ during progressive exercise. J of Appl Physiol. 1991;71:1076-108.

3. Jacobs I, Bleue S, Goodman J. Creatine ingestion increases anaerobic capacity and maximum accumulated oxygen deficit. Can J Appl Physiol. 1997;22:231-43.

4. Doherty M. The effects of caffeine on the maximal accumulated oxygen deficit and short-term running performance. Int J Sport Nutr. 1998;8:95-104.

5. Spriet LL. Anaerobic metabolism during high-intensity exercise. In: Hargreaves M, editor. Exercise metabolism. Champaign: Humans Kinetics; 1995. p. 1-39.

6. Margaria R, Edwards HT, Dill DB. The possible mechanisms of contracting and paying the oxygen debt and the role of lactic acid in muscular contraction. Amer J Physiol. 1933;106:689-715.

7. di Prampero PE, Ferretti G. The energetics of anaerobic muscle metabolism: a reappraisal of older and recent concepts. Respir Physiol. 1999;118:103-15.

8. Haseler LJ, Hogan MC, Richardson RS. Skeletal muscle phosphocreatine recovery in exercise-trained humans is dependent on O 2 availability. J of Appl Physiol. 1999;86:2013-8.

9. Piiper J, Spiller P. Repayment of $\mathrm{O}_{2}$ debt and resynthesis of high-energy phosphates in gastrocnemius muscle of the dog. J Appl Physiol. 1970;28:657-62.

10. Jansson E, Dudley GA, Norman B, Tesch PA. Relationship of recovery from intense exercise to the oxidative potential of skeletal muscle. Acta Physiol Scand. 1990;139:147-52.

11. Bogdanis GC, Nevill ME, Lakomy HK, Boobis LH. Power output and muscle metabolism during and following recovery from 10 and 20 s of maximal sprint exercise in humans. Acta Physiol Scand. 1998;163:261-72.

12. McMahon S, Jenkins D. Factors affecting the rate of phosphocreatine resynthesis following intense exercise. Sports Med. 2002;32:761-84.

13. Meyer T, Faude O, Scharhag J, Urhausen A, Kindermann W. Is lactic acidosis a cause of exercise induced hyperventilation at the respiratory compensation point? Br J Sports Med. 2004;38:622-5.

14. Marth PD, Woods RR, Hill DW. Influence of time of day on anaerobic capacity. Percept Mot Skills. 1998;86:592-94.

15. Howley ET, Basset DT, Welch HG. Criteria for maximal oxygen uptake: review and commentary. Med Sci Sports Exerc. 1995;27:1292-301.

16. Millet GP, Jaquen B, Borrani F, Candau R. Effects of concurrent endurance and strength training on running economy and $\mathrm{VO}_{2}$ kinetics. Med Sci Sports Exerc. 2002;34:1351-9. 
Silva-Cavalcante MD, et al.

17. Beneke R, Pollmann C, Bleif I, Leithäuser RM, Hütler M. How anaerobic is the Wingate anaerobic test for humans? Eur J Appl Physiol. 2002;87:388-92.

18. Beneke R, Beyer T, Jachner C, Erasmus J, Hütler M. Energetics of karate kumite. Eur J Appl Physiol. 2004;92:518-23.

19. Özyener F, Rossiter HB, Ward SA, Whipp BJ. Influence of exercise intensity on the on- and off-transient kinetics of pulmonary oxygen uptake in humans. J Physiol (Lond.). 2001;533:891-902.

20. Greenhaff PL, Timmons JA. Interaction between aerobic and anaerobic metabolism during intense muscle contraction. Exerc Sport Sci Rev. 1998;26:1-30.

21. Greenhaff PL. The creatine-phosphocreatine system: there's more than one song in its repertoire. J Physiol (Lond.). 2001;15(Pt 3):657.

22. Rossiter HB, Ward SA, Doyle VL, Howe FA, Griffiths JR, Whipp BJ. Inferences from pulmonary $\mathrm{O}_{2}$ uptake with respect to intramuscular [phosphocreatine] kinetics during moderate exercise in humans. J Physiol (Lond.). 1999;1(Pt 3):921-32.

23. Rossiter HB, Ward SA, Kowalchuck JM, Howe FA, Griffiths JR, Whipp BJ. Dynamic asymmetry of phosphocreatine concentration and $\mathrm{O}_{2}$ uptake between the on- and off-transients of moderate- and high-intensity exercise in humans. J Physiol (Lond.). 2002;15(Pt 3):991-1002.

24. Walsh B, Tiivel T, Tonkonogi M, Sahlin K. Increased concentrations of Pi and lactic acid reduce creatine-stimulated respiration in muscle fibers. J Appl Physiol. 2002;92:2273-6.

25. Grassi B. Delayed metabolic activation of oxidative phosphorylation in skeletal muscle at exercise onset. Med Sci Sports Exerc. 2005;37:1567-73.

26. Robergs RA, Ghiasvand F, Parker D. Biochemistry of exercise-induced metabolic acidosis. Am J Physiol Regul Integr Comp Physiol. 2004;287:R502-16.

27. Svedahl K, MacIntosh BR. Anaerobic threshold: the concept and methods of measurement. Can J Appl Physiol. 2003:28:299-323.

28. Bertuzzi RC, Franchini E, Ugrinowitsch C, et al. Predicting MAOD using only a supramaximal exhaustive test. Int J Sports Physiol Perform. 2010;31:477-81.

29. Artioli GG, Bertuzzi RC, Roschel H, Mendes S H, Lancha Junior AH, Franchini E. Determining the contribution of the energy systems during exercise. J Vis Exp. 2012;61:e3413.

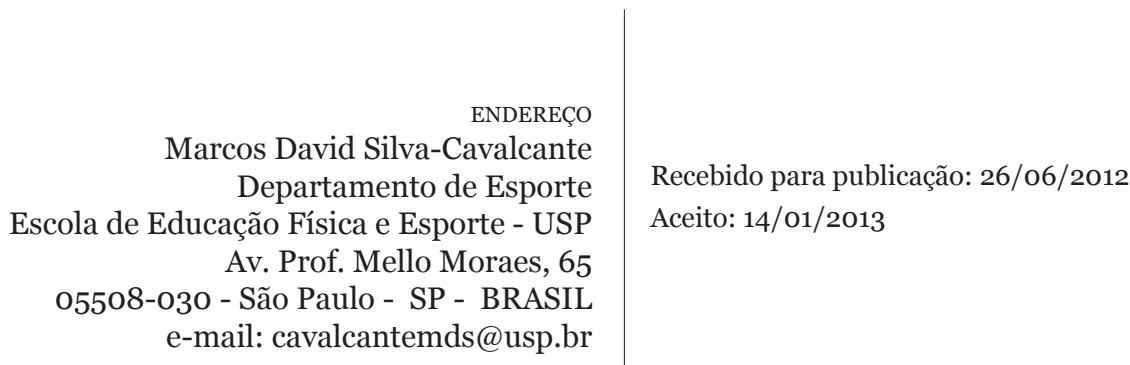

216 • Rev Bras Educ Fís Esporte, (São Paulo) 2013 Abr-Jun; 27(2):209-16 